\title{
Secondary mania - from brain lesions to functional connectivity
}

Gonçalo Cotovio, MD*; Daniel Talmasov, MD*; J. Bernardo Barahona-Corrêa, MD, PhD; Joey Hsu, Suhan Senova, MD, PhD; Ricardo Ribeiro Msc; Louis Soussand, Msc; Ana Velosa, MD; Natalia Rost, MD; Ona Wu, PhD; Alexander Cohen, MD, PhD; Albino J. Oliveira-Maia, MD, MPH, PhD\#; Michael D. Fox, MD, PhD\#

Champalimaud Research \& Clinical Centre, Champalimaud Centre for the Unkown, Lisboa, Portugal

Berenson-Allen Center for Non-Invasive Brain Stimulation, Beth Israel Deaconess Medical Center, Harvard Medical School, Boston, MA, USA Department of Psychiatry and Mental Health, Centro Hospitalar de Lisboa Ocidental, Lisboa, Portugal NOVA Medical School | Faculdade de Ciências Médicas, Universidade Nova de Lisboa, Lisboa, Portugal

Background and aims: Mania, a neuropsychiatric syndrome regarded as hallmark of Bipolar Disorder, can occur as a consequence of focal brain lesions. Such cases may provide insight into the regions responsible for manic-symptoms. However, mania has been reported following lesions in multiple different brain regions, leaving the neuroanatomical substrate unclear.

Objective: To investigate whether lesion-locations associated with mania share a common pattern of brain connectivity.

Brain lesions associated with new-onset mania were identified from two sources: published case-reports $(\mathrm{N}=46)$ and a clinical retrospective-cohort $(\mathrm{N}=15)$.
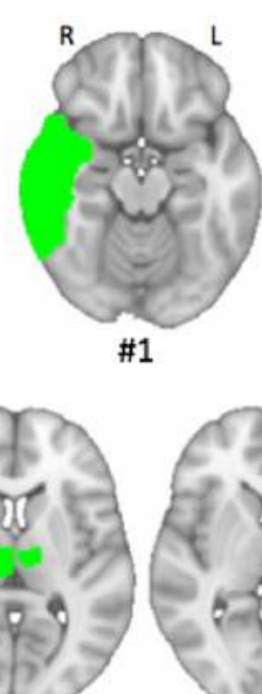

\#6
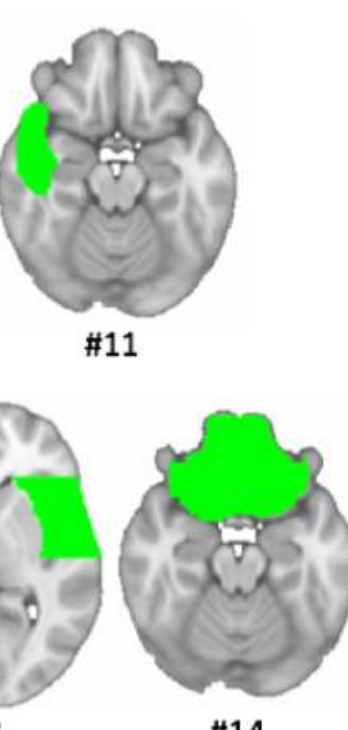

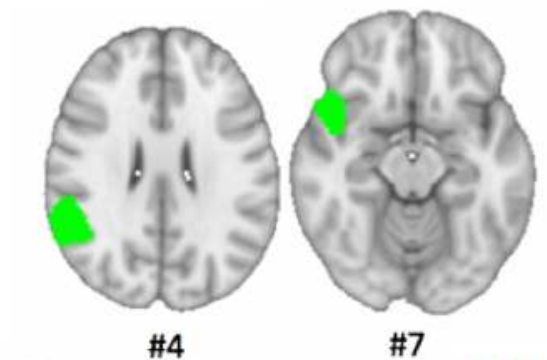

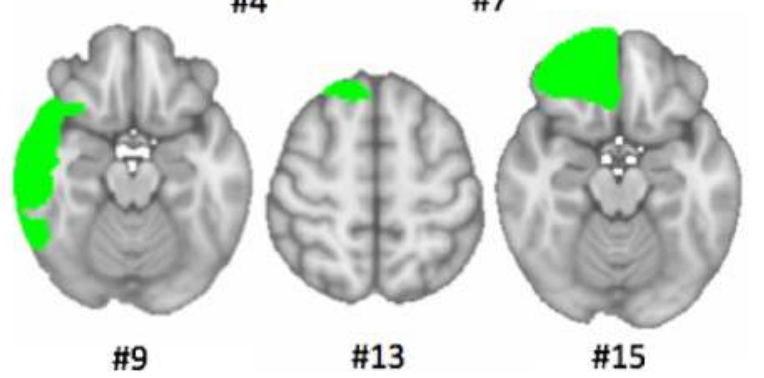

3D mania lesions (green) encompass ROls in the 2D Functional Connectivity Map that are more associated with mania
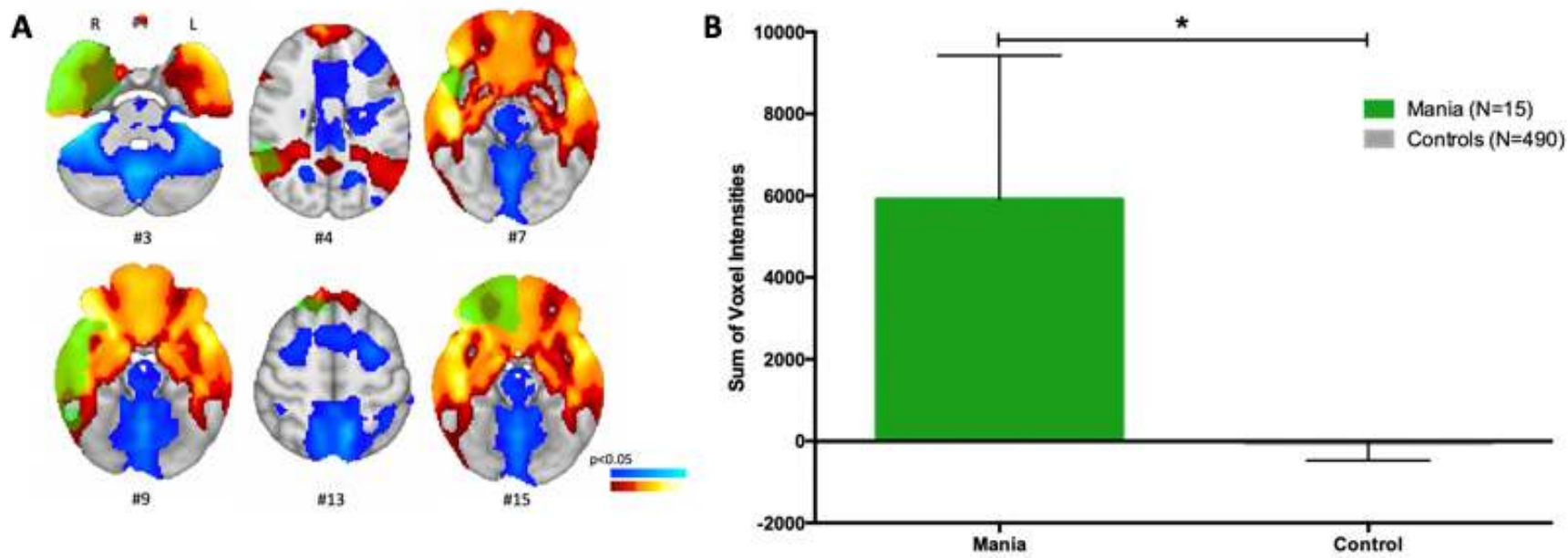

Results were independent of lesion-etiology and bipolar risk factors.
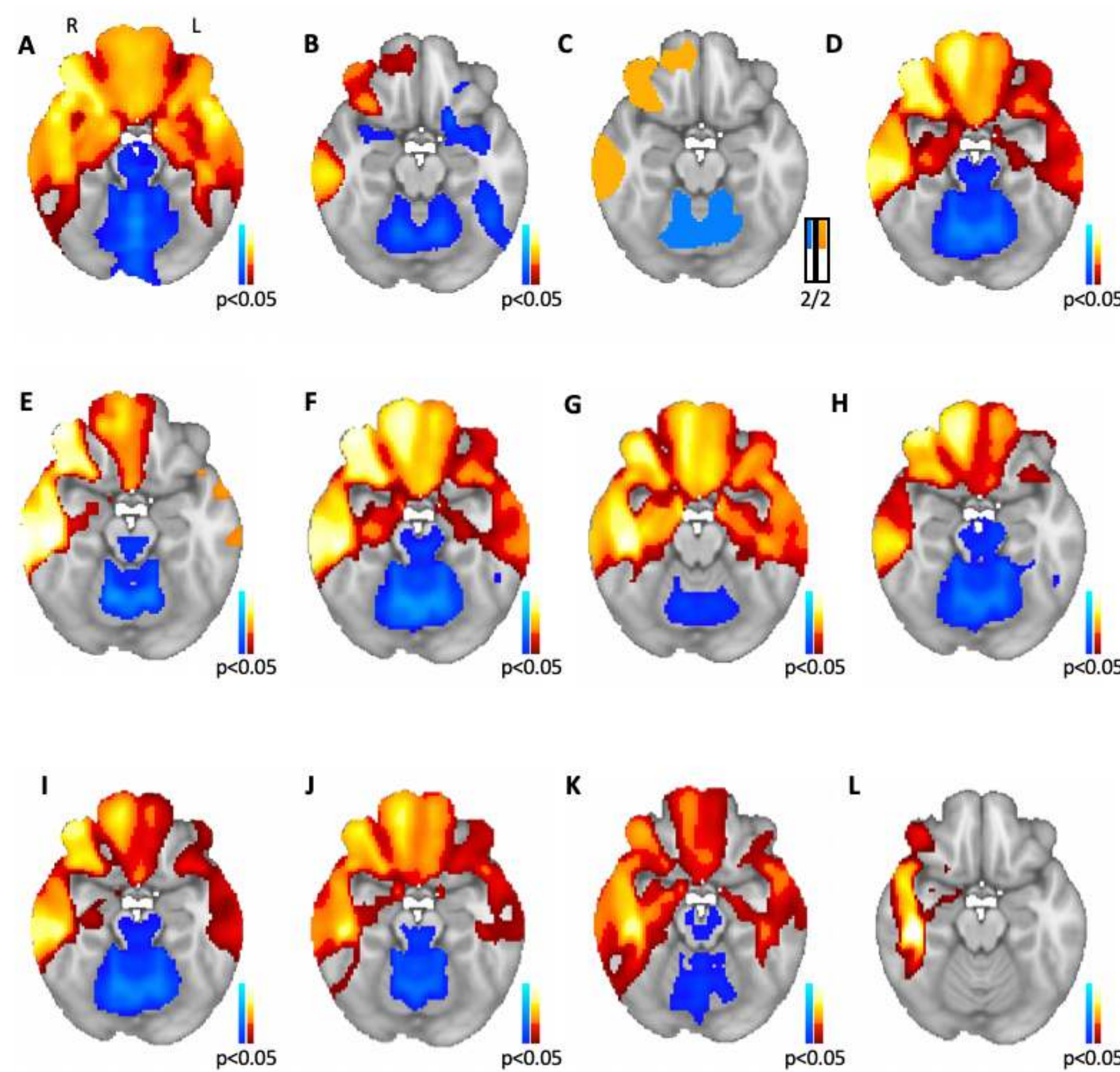

A - 2D literature-based cohort

B - Replicated in our 3D clinical cohort

G - Higher likelihood of association

$\mathrm{H}$ - Lower likelihood of association

C - Conjunction analysis

I - All core mania symptoms

$\mathrm{J}$ - No mania risk factors

$\mathrm{K}$ - Only ischemic stroke

E - Full cohort with extra 2D controls

F - Full cohort controlling for lesion size

\section{Conclusions}

Lesions associated with mania occur in different brain locations, but share connectivity to specific frontal, temporal and thalamic area, lending insight into the functional neuroanatomy of mania, providing hypotheses for investigation of brain abnormalities in bipolar patients, and identifying potential therapeutic targets.

* Shared first authors 